\title{
A dynamic box model to predict the radionuclide behaviour in rivers for medium and long-term periods
}

\author{
P. Boyer ${ }^{1}$, K. Beaugelin-Seiller ${ }^{1}$, F. Ternat ${ }^{1}$, F. Anselmet ${ }^{2}$ and M. Amielh ${ }^{2}$ \\ ${ }^{1}$ IRSN, DEI, CE Cadarache, BP. 3, 13115 St. Paul-Lez-Durance, France \\ ${ }^{2}$ IRPHE, Technopôle Château-Gombert, BP. 146, Marseille, France
}

\begin{abstract}
This paper presents a dynamic box model for the radionuclide behaviour in rivers on medium- and long-term periods (several days to several years). The river is described as a succession of boxes representative of its different reaches. In each reach, the compartments are the water column and three bottom sediment layers. Called interface, the first layer plays a fundamental role for the vertical exchanges of solid radionuclide phases between the water column and the sediment. The second layer results from the consolidation of the previous one. Its interstitial water is mobile and the dissolved radionuclide phases can be exchanged with the water column. It is called active. The last layer results from the consolidation of the active layer. Its interstitial water is slightly mobile and it is assumed that its dissolved radionuclide phases cannot be exchanged. It is called passive. In each compartment, the model computes the temporal evolution of the radionuclide activities in the main abiotic and biotic components. The abiotic components are the water and different matter classes classified according to their deposit kinetics. The biotic components are phytoplankton, zooplankton, and fish distributed in planktonivorous and omnivorous species, in water column and macrobenthos in bottom sediment.
\end{abstract}

\section{INTRODUCTION}

This paper presents a dynamic box model to study and predict the radionuclide behaviour in rivers for medium- and long-term periods (from several days to several years). This model is implemented in the version 0.1 of the CASTEAUR code, the version 0.0 being dedicated to short-term assessments mainly associated to accidental situations [1]. For long-term periods, the sediment plays a fundamental role for the radionuclide transfers [2]. In function of the local and hydraulic conditions, this compartment can accumulate (deposition), rapidly be released (flood event), slowly exchange (diffusion) or definitively stock (consolidation) radionuclides. In this context, and after more than 50 years of military and civil nuclear activities, it seems important to assess the constituted stocks and their capacity to be remobilised, in associating modelling and in-situ measurements. Compared to equivalent models for long-term assessments [3-4], the approach proposed in this paper is based on a strong coupling between hydraulics, sedimentary dynamics and radionuclide transfers.

Because it is well known now that radionuclide speciation is depending on granulometry and the nature of matters [5-6], the model can take into account several matter classes identified and classified according to their settling kinetics [7]. In the bottom sediment, three main layers are classically considered to compute radionuclide transfers in rivers [8]: an interface, an active layer and a passive layer. The present approach proposes specific sedimentary modelling for the interface layer taking into account several classes of particles and it introduces the effects of compaction processes on vertical transfers of the solid and dissolved radionuclide phases.

Always in the context of long-term assessments, the filiation processes could become non-negligible for some radionuclides. These processes are also included in the model. 
The model is built upon five sub-models, related to: 1) hydrography, 2) hydraulics, 3) sedimentary dynamics, 4) trophic chain and 5) radioecology. In comparison to CASTEAUR v0.0, the main evolutions concerns the hydraulic, sedimentary and radioecological models. As the trophic chain model is not much changed, it will not be presented again here (see [1]).

\section{GENERAL CONSIDERATIONS}

A box approach is considered, the boxes being identified to the river reaches ( $\S 3$ ). Consequently, the results and the parameters are assumed uniform at the scale of a reach. This point limits the domain of application to time steps equivalent to the hydraulic characteristic times of the domain (typically, one to several days). The calculations give the temporal evolution of the radionuclide stocks in the different components.

\section{HYDROGRAPHIC AND HYDRAULIC MODELS}

The hydrographic model describes the geometry of the river. It is based on a succession of reaches, constituting a linear hydrographic network. The main criterion for identifying a reach is the part of the river limited by two inflows. On a reach, the hydrographic parameters are the length $L_{b}(\mathrm{~m})$, the average slope $I_{b}\left(\mathrm{~m} . \mathrm{m}^{-1}\right)$ and the trapezium bathymetric section form (figure 1).

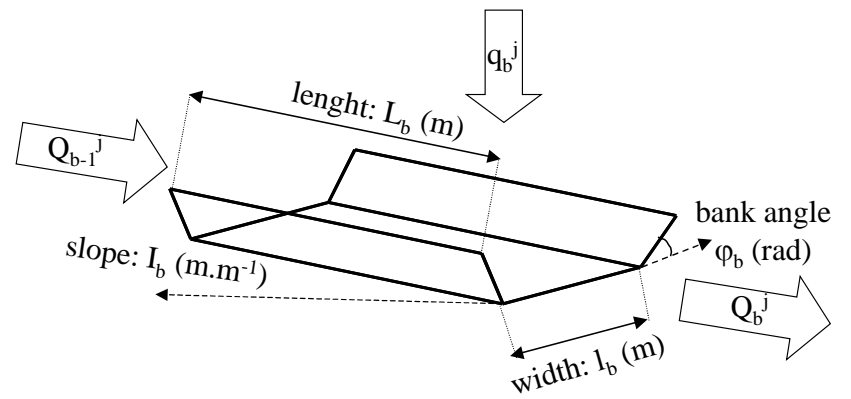

Figure 1. Hydrographic parameters and water flow model.

The hydraulic model assesses the temporal evolutions of the water flow $Q_{b}{ }^{j}\left(\mathrm{~m}^{3} . \mathrm{s}^{-1}\right)$, the water column volume $w c_{b}{ }^{j}\left(\mathrm{~m}^{3}\right)$ and the shear stress of the flow on the bottom $\tau_{b}^{j}\left(\mathrm{~N} \cdot \mathrm{m}^{-2}\right)$. These results are used in the sedimentary ( $\S 4)$ and the radioecological models ( $\S 5$ ) to compute the fluxes of matter and radionuclides between the reaches and between the column and the sediment. The temporal evolutions of the water column stocks are calculated by:

$$
\frac{d w c_{b}^{j}}{d t}=Q_{b-1}^{j}+q_{b}^{j}-Q_{b}^{j}
$$

$q_{b}^{j}\left(\mathrm{~m}^{3} \cdot \mathrm{s}^{-1}\right)$, the water inflow in the reach $b$ for the day $j$, is an input data.

Considering $Q_{b}^{j}=U_{b}^{j} \cdot S M_{b}^{j}$ and the Strickler relation for uniform flow conditions [9], $U_{b}^{j}=K s_{b} \cdot R h_{b}^{j \frac{2}{3}} \cdot \sqrt{I_{b}}$, the water depth, and consequently the bottom shear stress, can be deduced [10]:

$$
\tau_{b}=I_{b} \cdot \rho \cdot g \cdot R h_{b}
$$

$S M_{b}^{j}$ is the wetted area $\left(\mathrm{m}^{2}\right), R h_{b}{ }^{j}$ is the hydraulic radius (m), $U_{b}^{j}$ is the flow mean speed $\left(\mathrm{m} . \mathrm{s}^{-1}\right)$ and $K s_{b}$ is the Strickler coefficient $\left(\mathrm{m}^{1 / 3} \cdot \mathrm{s}^{-1}\right)$. 


\section{SEDIMENTARY MODEL}

The sedimentary model assesses the stocks and the fluxes of solid matter needed for the calculation of radionuclide transfers both under solid and dissolved forms ( $\S 5$ ). Three sedimentary layers are considered. The first one is an interface layer with the water column. It is a very fine layer constituted of recent deposit not compacted yet [11]. So, it can be admitted, in this layer, that all the matters have a non-cohesive behaviour. Its associated parameters are $h_{m a x}(\mathrm{~m})$, its maximal thickness (typically, few times the matter diameter) and $\phi_{i l}(\%)$, its mean water content $(\approx 80 \%)$. The second layer results from the compaction of the interface. If the matter is cohesive, the consolidation is initiated but the interstitial water remains mobile and the dissolved phases can be exchanged with the column. This is the active layer, parameterised by $\operatorname{hmax}_{a l}(\mathrm{~m})$, its maximal thickness (some centimeters), and $\phi_{a l}(\%)$, its mean water content $(\approx 60 \%)$. The compaction of the active layer induces the third layer, where the consolidation becomes strong enough to reduce the mobility of interstitial water, and the exchanges of dissolved radionuclide phases are negligible. This layer is called the passive layer. It is parameterised only by $\phi_{p l}(\%)$, its mean water content $(\approx 40 \%)$.

In each reach $b$, for each particle class $m$, and for each time step $j$, the sedimentary model computes the masses $(\mathrm{kg}) \underset{w c}{m_{b}^{j}}$, in the water column, $m_{b}^{j}$, in the interface layer, $m_{b}^{j}$, in the active layer, and $\underset{b l}{m_{b}^{j}}$, in the passive layer.

In the water column, $w_{b c}^{j}\left(\mathrm{~m}^{3}\right)$, the water volume, is assimilated to $w c_{b}^{j}$; when in interface, active and passive layers, water volumes are respectively given by:

$$
w_{b}^{j}=\frac{1-\Phi_{i l}}{\Phi_{i l}} \cdot \frac{M_{b}^{j}}{\rho_{w}} ; w_{b}^{j}=\frac{1-\Phi_{a l}}{\Phi_{a l}} \cdot \frac{M_{b l}^{j}}{\rho_{w}} ; w_{b l}^{j}=\frac{1-\Phi_{i l}}{\Phi_{p l}} \cdot \frac{M_{b l}^{j}}{\rho_{w}}
$$

where $\rho_{w}\left(\mathrm{~kg} \cdot \mathrm{m}^{-3}\right)$ is the water density and $M_{b}^{j}, M_{b}^{j}$ and $\underset{\substack{b \\ \text { il }}}{M_{b}^{j}}$ are the total masses of these layers. Figure 2 presents the different fluxes considered in this sedimentary model.

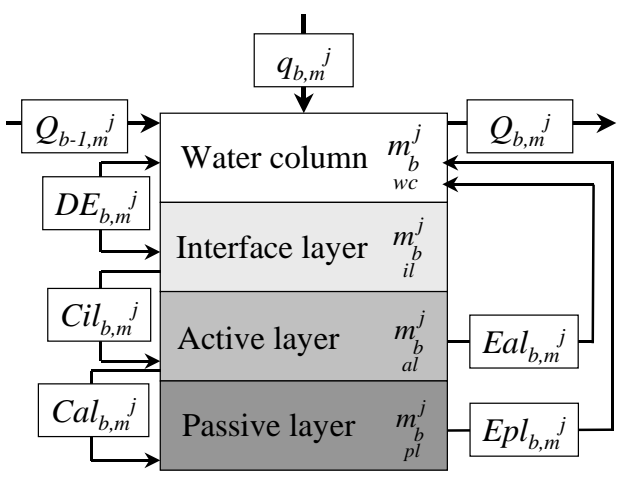

Figure 2. Sedimentary fluxes $\left(\mathrm{kg} \cdot \mathrm{s}^{-1}\right)$.

- $\quad Q_{b-1, m}^{j}$ and $Q_{b, m j}$ are the entering flux, coming from the upstream reach, and the outgoing flux.

$$
Q_{b-1, m}^{j}=Q_{b-1}^{j} \cdot \underset{w c}{[m]_{b-1}^{j}}=Q_{b-1}^{j} \cdot \frac{m_{b-1}^{j}}{w c_{b-1}^{j}} \text { and } Q_{b, m}^{j}=Q_{b}^{j} \cdot \underset{w c}{[m]_{b}^{j}}=Q_{b-1}^{j} \cdot \frac{m_{b c}^{j}}{w c_{b}^{j}}
$$


- $\quad q_{b, m}{ }^{j}$ is an entering flux coming from outside the study domain. It is an input data.

- $\quad D E_{b, m}{ }^{j}$ is the balance, for the particle class $m$, between erosion and deposition fluxes related to the water column and the interface layer. In the latter, as the matter is supposed not to be consolidated, the balance is applied to each class independently of the other ones. Its formulation is given by [12]:

$$
\left.D E_{b, m}^{j}=\frac{W c_{m}}{R h_{b}^{j}} \cdot \underset{\substack{w c \\ w c}}{\left(m_{b}^{j}-m_{b}^{j^{*}}\right.}\right)
$$

$m_{b c}^{j^{j^{*}}}$, the equilibrium mass of class $\mathrm{m}$ in the water column, is depending on the matter and the hydraulic conditions. It is given by:

$$
m_{w c}^{j^{*}}=\frac{e_{m}}{W c_{m}} \cdot\left(\frac{\tau_{b}^{j}}{\tau c e_{m}}-1\right) \cdot w c_{b}^{j} \text { if } \tau_{b}^{j}>\tau c e_{m} \text { and } m_{w c}^{j^{*}}=0 \text { if } \tau_{b}^{j} \leq \tau c e_{m}
$$

For each class $m, W c_{m}\left(m . s^{-1}\right)$ is the settling velocity, $\tau c e_{m}\left(N \cdot m^{-2}\right)$, the critical erosion shear stress, and $e_{m}\left(\mathrm{~kg} \cdot \mathrm{m}^{-2} \cdot \mathrm{s}^{-1}\right)$, the erosion rate. These parameters can be theoretically computed or experimentally measured from experimental devices [7-12].

- $\quad \mathrm{Cil}_{b, m}{ }^{j}$ and $\mathrm{Cal}_{b, m}{ }^{j}$ are the matter fluxes resulting from the compaction of the interface and the active layer. When the thickness of a layer becomes larger than its maximal value, the matter in excess is transferred to the inferior layer. At this stage, the cohesive behaviour of the matter has to be taken into account. Then, these transfers do not concern any more the classes individually but their melange. The transfers by class are deduced according to the contribution of each class to the melange.

- $E a l_{b, m}{ }^{j}$ and $E p l_{b, m}{ }^{j}$ are the erosion fluxes of the active and passive layers. For each one, these fluxes are activated only if the superior layer has been totally eroded. As for compaction, the cohesive behaviour of the matter has to be taken into account: these fluxes, modelled with the approach of Partheniades [13], are applied to the melange. The transfers by class are already known, according to the contribution of each class to the melange.

\section{RADIOECOLOGICAL MODEL}

The radioecological model uses the results provided by the previous models to compute the radionuclide activities $(\mathrm{Bq})$. For each time step $j$ and each reach $b$, the targets are the radionuclide activities under dissolved and solid phases, noted $\underset{\substack{b, w \\ w c}}{r^{j}}$ and $\underset{\substack{b, m \\ w c}}{r_{j}^{j}}$, in the water column, $\underset{\substack{b, w \\ \text { il }}}{r_{j}^{j}}$ and $\underset{\substack{b, m \\ i l}}{j}$, in the interface layer, $\underset{\substack{b, w \\ a l}}{r^{j}}$ and $\underset{\substack{b, m \\ a l}}{j}$, in the active layer, and $\underset{\substack{b, w \\ p l}}{r_{j}^{j}}$ and $\underset{\substack{b, m \\ p l}}{r^{j}}$, in the passive layer.

Considering the temporal scale resolution and the small thickness of the interface layer, the equilibrium hypothesis between this layer and the water column is assumed. Four kinds of radionuclide fluxes are taken into account: 1) between reaches, 2) between components, 3) between solid and dissolved phases and 4) through radioactive decay and the resulting filiations.

\subsection{Fluxes between reaches and between components}

Between reaches and between components, the radionuclide fluxes, associated to solid and dissolved phases, are respectively presented on figures $3 a$ and $3 b$. 


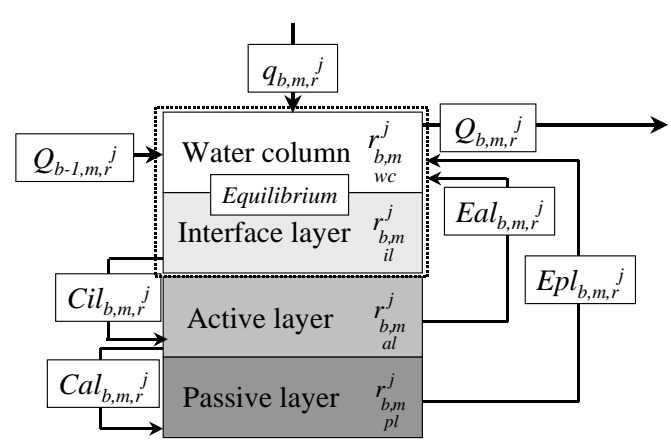

a)

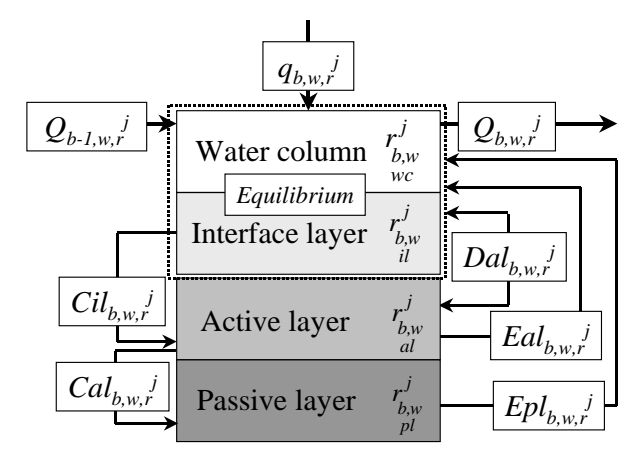

b)

Figure 3. Solid (a) and dissolved (b) radionuclide fluxes (Bq.s ${ }^{-1}$ ) between reaches and between components.

Considering the generic notations $\mathrm{cmp}$ for component and $F$ for flux, all the radionuclide fluxes - for both the solid and dissolved phases associated to the hydraulic and sedimentary processes

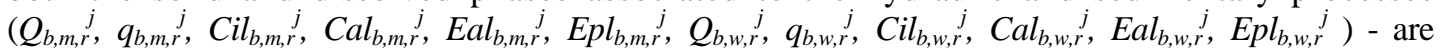
directly given by:

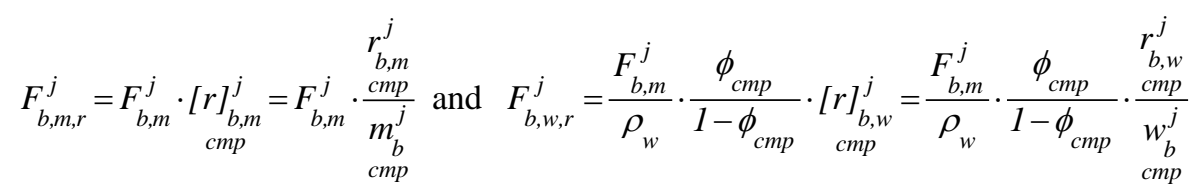

- $\quad q_{b, m, r}^{j}$ and $q_{b, w, r}^{j}$, the entering fluxes coming from outside the study domain, are input data.

- $\quad \operatorname{Dal}_{b, w, r}{ }^{j}$ is a diffusing flux of the dissolved phase between the active layer and the water column. Its formulation is:

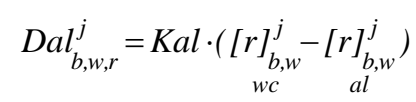

$\mathrm{Kal}\left(\mathrm{m}^{3} \cdot \mathrm{s}^{-1}\right)$ is the exchange flow between the water column and the active layer.

\subsection{Fluxes between solid and dissolved phases}

The time steps (one to several days) are long enough to apply an equilibrium hypothesis to these fluxes [14]. Under this consideration, the total activity of the radionuclide $r$ in a component $c m p$ is a variable which becomes independent from these exchanges because it is given by:

$$
\substack{b \\ c p m}_{\substack{b, w \\ c m p}}^{j}+\sum_{m}^{j} r_{\substack{b, m \\ c m p}}^{j}
$$

Excepted for the water column and the interface layer, the equilibrium hypothesis allows one to write:

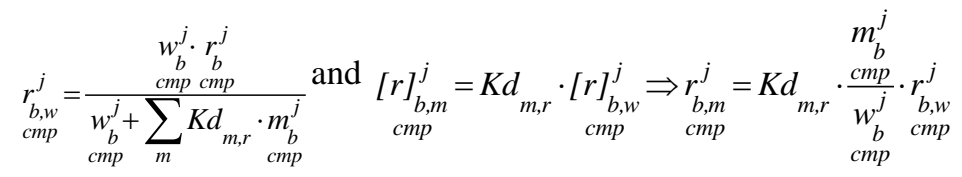

$K d_{m, r}\left(\mathrm{~m}^{3} \cdot \mathrm{kg}^{-1}\right)$ is the equilibrium coefficient of the radionuclide $r$ between its dissolved phase and its solid phase associated to the class $m$. A protocol is presently studied at IRSN to determine this parameter [7]. 
The equilibrium assumption for the radionuclide concentrations between the interface layer and the water column involves to resolve these exchanges for a same single global compartment, wcil, assembling the water column and the interface layer. The relations (9) and (10) are then applied to the following sums:

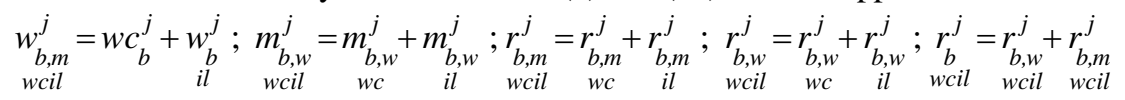

The dissolved and solid activities in the water column and the interface layer are then given by:

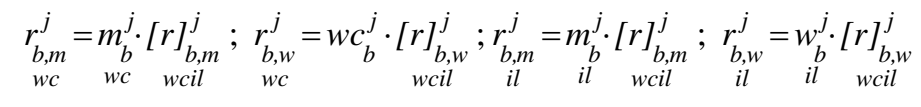

\subsection{Fluxes by radioactive decay and filiations}

These fluxes transfer a part of the activity of a radionuclide to another one in the same phase and the same component. For a radionuclide $r$, the parameters are $\lambda_{r}\left(\mathrm{~s}^{-1}\right)$, its decay constant and the list of its mother's $p$ if the filiations are activated. Typically, the set of equations specific to the resolution of these fluxes is:

$$
\frac{d r_{b}^{j}}{d t}=-\lambda_{r} \cdot \underset{c m p}{r_{b}^{j}}+\sum_{p} \lambda_{p} \cdot \underset{c m p}{p_{b}^{j}}
$$

\section{CONCLUSION AND PERSPECTIVES}

This paper presented a dynamic box model which computes the radionuclide behaviour in rivers for medium- and long-term periods with a daily time step. Implemented in the version 0.1 of the CASTEAUR code, coupling between hydraulic, sedimentary dynamic and radionuclide transfers is proposed to refine the consideration of the main processes that strongly influence the transfers of the solid and dissolved radionuclide phases for medium- or long-term periods: transport in the water column, deposit of solid phases, erosion of solid phases, diffusion of the dissolved phase between the sediment and the water column, consolidation of the sediment layers. Particularly, the sedimentary model is based on several classes of matter identified according to their settling kinetics and three bottom sediment layers (interface, active, passive).

Finally, for some radionuclides and for long-term assessments, the filiation processes could become non-negligible and they are consequently also included in the model.

The next development will be a coupling with a dosimetric model to compute dose assessments. This model is the first step of a current thesis work devoted to the refinement of the characterization and the modelling of the bottom sediment processes (particularly, the consolidation ones) and their resulting influences on the radionuclide transfers.

\section{References}

[1] Duchesne S., Boyer P. and Beaugelin-Seiller K., "Sensitivity and uncertainty analysis of a model computing radionuclides transfers in fluvial ecosystems (CASTEAUR): application to ${ }^{137} \mathrm{Cs}$ accumulation in chubs" Ecological Modelling 166 (2003) 257-276.

[2] Eyrolle F., Charmasson S. and Louvat D., "Plutonium isotopes in the lower reaches of the River Rhône over the period 1945-2000: fluxes towards the Mediterranean Sea and sedimentary inventories" J. Environ. Radioactivity 74 (2004) 127-138.

[3] Monte L. "A collective model for predicting the long-term behaviour of radionuclides in rivers" Sci. Total Environ. 201 (1997) 17-29. 
[4] Smith J.T., Fesenko S.V., Howard B.J., Horrill A. D., Sanzharova N.I., Alexakhin R.M., Elder D.G. and Naylor, C., "Temporal Change in Fallout 137Cs in Terrestrial and Aquatic Systems: A Whole Ecosystem Approach" Environ. Sci. Technol. 33 (1999) 49-54.

[5] Borovec, Z., "Element in size-fractionated bottom sediment of the Elbe River in its Czech part" Aquat. Sci. 62 (2000) 232-251.

[6] Vray F., Debayle C. and Louvat D., "Long-term flux of Chernobyl-derived ${ }^{137}$ Cs from soil to French rivers: a study on sediment and biological inidicators" J. Environ. Radioactivity 68 (2003) 93-114.

[7] Brach-Papa C., Boyer P., Amielh M. and Anselmet F., "Characterization and radionuclides sorption of suspended particulate matters in freshwater according to their settling kinetics" Radioprotection 39 (Suppl. 1) (2004).

[8] Håkanson L. and Monte L. In Modelling radioactivity in the environment (Elsevier, Edited by Scott E.M., 2003) pp. 147-200.

[9] Graf W.H. and Altinakar M.S., Hydraulique fluviale - Écoulement et phénomènes de transport dans les canaux à géométrie simple (Presses Polytechniques et Universitaires Romandes, Lausanne, 2000) pp. 71-87.

[10] Viollet P.L., Chabard J.P., Esposito P. and Laurence D., Mécanique des fluides appliquée (Presses de l'École Nationale des Ponts et Chaussées, Paris, 1998) p 230.

[11] Maa, J.P., Sanford, L. and Halka, J.P., "Sediment resuspension characteristics in Baltimore Harbour, Maryland" Marine Geology, 146 (1998). 137-145.

[12] El Ganaoui O., Schaaf E., Boyer P., Amielh M., Anselmet F. and Grenz C., "The deposition and erosion of cohesive sediments determined by a multi-class model" Estuarine, Coastal and Shelf Science 60 (2004) 457-475.

[13] Partheniades E., "Erosion and deposition of cohesive soils" Journal of Hydraulics Division (ASCE) 91 (1965) 105-139.

[14] Beaugelin-Seiller K., "Influence des temps d'atteinte de l'équilibre pour les transferts de radionucléides traités par CASTEAUR sur le choix d'un modèle moyen long terme pour la chaîne trophique" Rapport IRSN/DEI - SECRE 03-016 (2003). 